Trinity College

Trinity College Digital Repository

Faculty Scholarship

2003

On Regular Graphs Optimally Labeled with a Condition at Distance Two

John P. Georges

Trinity College, john.georges@trincoll.edu

David W. Mauro

Trinity College, david.mauro@trincoll.edu

Follow this and additional works at: https://digitalrepository.trincoll.edu/facpub

Part of the Mathematics Commons 


\title{
ON REGULAR GRAPHS OPTIMALLY LABELED WITH A CONDITION AT DISTANCE TWO*
}

\author{
JOHN P. GEORGES ${ }^{\dagger}$ AND DAVID W. MAURO ${ }^{\dagger}$
}

\begin{abstract}
For positive integers $j \geq k$, the $\lambda_{j, k}$-number of graph $G$ is the smallest span among all integer labelings of $V(G)$ such that vertices at distance two receive labels which differ by at least $k$ and adjacent vertices receive labels which differ by at least $j$. We prove that the $\lambda_{j, k}$-number of any $r$-regular graph is no less than the $\lambda_{j, k}$-number of the infinite $r$-regular tree $T_{\infty}(r)$. Defining an $r$-regular graph $G$ to be $(j, k, r)$-optimal if and only if $\lambda_{j, k}(G)=\lambda_{j, k}\left(T_{\infty}(r)\right)$, we establish the equivalence between $(j, k, r)$-optimal graphs and $r$-regular bipartite graphs with a certain edge coloring property for the case $\frac{j}{k}>r$. The structure of $r$-regular optimal graphs for $\frac{j}{k} \leq r$ is investigated, with special attention to $\frac{j}{k}=1,2$. For the latter, we establish that a $(2,1, r)$-optimal graph, through a series of edge transformations, has a canonical form. Finally, we apply our results on optimality to the derivation of the $\lambda_{j, k}$-numbers of prisms.
\end{abstract}

Key words. $L(j, k)$-labeling, regular graph, prism

AMS subject classification. 05C

DOI. $10.1137 / \mathrm{S} 0895480101391247$

1. Introduction. For positive integers $j$ and $k$ with $j \geq k$, an $L(j, k)$-labeling of graph $G$ is an assignment $L$ of nonnegative integers to the vertices of $G$ such that

(1) $|L(v)-L(u)| \geq j$ if $v$ and $u$ are adjacent, and

(2) $|L(v)-L(u)| \geq k$ if $v$ and $u$ are distance two apart.

Elements of the image of $L$ are called labels, and the span of $L$, denoted $s(L)$, is the difference between the largest and smallest labels. The minimum span taken over all $L(j, k)$-labelings of $G$, denoted $\lambda_{j, k}(G)$, is called the $\lambda_{j, k}$-number of $G$, and if $L$ is a labeling with minimum span, then $L$ is called a $\lambda_{j, k}$-labeling of $G$. Unless otherwise stated, we shall assume with no loss of generality that the minimum label of $L(j, k)$-labelings of $G$ is 0 .

A variation of Hale's channel assignment problem [12], the problem of labeling a graph with a condition at distance two, was first investigated in the case $j=2$ and $k=1$ by Griggs and Yeh [11]. Other authors have since explored the $\lambda_{2,1}$-numbers of graphs in various classes, as well as relationships between $\lambda_{2,1}(G)$ and other invariants of $G$ (see $[2,6,9,10,13,14,16,17,18,19])$. Additionally, properties of $\lambda_{j, k}$-numbers have been investigated in $[1,4,5]$ and $[7]$.

In this paper, we develop the notion of optimality among $r$-regular graphs by considering the $\lambda_{j, k}$-number of the infinite $r$-regular tree $T_{\infty}(r), r \geq 2$ [4]. We show in section 2 that $\lambda_{j, k}(G) \geq \lambda_{j, k}\left(T_{\infty}(r)\right)$ for any $r$-regular graph $G$, and we define $G$ to be $(j, k, r)$-optimal if and only if the equality holds. In section 3 , we consider the structure of $(j, k, r)$-optimal graphs for $\frac{j}{k}>r$ and show that $(j, k, r)$-optimal graphs are bipartite with block edge coloring number $r$. In section 4 , we define the notion of cyclic optimality in the exploration of the case $\frac{j}{k} \leq r$, with special attention to $j=k=1$. We consider the structure of $(2,1, r)$-optimal graphs in section 5 and

\footnotetext{
${ }^{*}$ Received by the editors June 15, 2001; accepted for publication (in revised form) May 26, 2003; published electronically November 14, 2003.

http://www.siam.org/journals/sidma/17-2/39124.html

†Department of Mathematics, Trinity College, Hartford, CT 06106 (john.georges@trincoll.edu, David.Mauro@trincoll.edu).
} 
establish a canonical form for such graphs. Finally, in section 6 , we use the results in the preceding sections to determine the $\lambda_{2,1}$-numbers and $\lambda_{1,1}$-numbers of prisms.

2. Definitions and preliminary results. Throughout the paper, $x \equiv y(\bmod$ $n$ ) shall mean that $x-y$ is divisible by $n$, and $x=y(\bmod n)$ shall mean that $x$ is set equal to the remainder that results when $y$ is divided by $n$.

Let $G$ be a graph and let $L$ be an $L(j, k)$-labeling of $G$. Then $M_{i}(G, L)=$ $\{v \in V(G) \mid L(v)=i\}$ and $m_{i}(G, L)=\left|M_{i}(G, L)\right|$. When there is no possibility of confusion, reference to $G$ and $L$ will be suppressed.

Georges and Mauro [4] derived $\lambda_{j, k}\left(T_{\infty}(r)\right)$ for all $j, k$, and $r$, including the two particular cases which will be of importance to this paper.

TheOREM 2.1. For $\frac{j}{k} \geq r, \lambda_{j, k}\left(T_{\infty}(r)\right)=j+(2 r-2) k$.

THEOREM 2.2 .

$$
\lambda_{j, 1}\left(T_{\infty}(r)\right)=\left\{\begin{array}{l}
r+2 j-2 \text { if } j \leq r, \\
j+2 r-2 \text { if } j \geq r .
\end{array}\right.
$$

We next show that $\lambda_{j, k}\left(T_{\infty}(r)\right)$ is a lower bound for the $\lambda_{j, k}$-numbers of all $r$ regular graphs, which in turn will serve to motivate the notion of $(j, k, r)$-optimality.

THEOREM 2.3. If $G$ is a connected $r$-regular graph, then $\lambda_{j, k}(G) \geq \lambda_{j, k}\left(T_{\infty}(r)\right)$.

Proof. Suppose $L$ is an $L(j, k)$-labeling of $G$ with span $s(L)$. It suffices to show that $L$ induces an $L(j, k)$-labeling of $T_{\infty}(r)$ with span $s(L)$.

Let $v_{n_{0}}$ be an arbitrarily selected vertex in $V(G)$ and let the neighbors of $v_{n_{0}}$ be $v_{n_{1}}, v_{n_{2}}, \ldots, v_{n_{r}}$. We assign the label $L\left(v_{n_{0}}\right)$ to the root $w_{0}$ of $T_{\infty}(r)$, and we assign the labels $L\left(v_{n_{1}}\right), L\left(v_{n_{2}}\right), \ldots, L\left(v_{n_{r}}\right)$ to the children $w_{1}, w_{2}, \ldots, w_{r}$ of $w_{0}$, respectively. The $r-1$ children of $w_{i}$ may then be assigned the labels of the neighbors of $v_{n_{i}}$ which have not already been assigned to the parent of $w_{i}$. The result follows by induction.

For the case $(j, k)=(2,1)$ and $r \geq 2$, the well-known inequality $\lambda_{2,1}(G) \geq r+2$ was used by Jha [13] in his consideration of the $\lambda$-number of the Kronecker product of cycles. There, he called those products with $\lambda_{2,1}$-numbers equal to the lower bound optimal. We extend his terminology to the consideration of optimal $(j, k, r)$-labelings of $r$-regular graphs as follows.

Definition 2.4. For $r \geq 2$, the graph $G$ is said to be $(j, k, r)$-optimal if and only if $G$ is r-regular and $\lambda_{j, k}(G)=\lambda_{j, k}\left(T_{\infty}(r)\right)$. If $L$ is a $\lambda_{j, k}$-labeling of $a(j, k, r)$ optimal graph $G$, then $L$ is said to be a $(j, k, r)$-optimal labeling of $G$. We denote the set of $(j, k, r)$-optimal graphs by $\Gamma(j, k, r)$.

It follows from Theorems 2.1 and 2.2 that $G$ is $(j, 1, r)$-optimal if and only if $\lambda_{j, 1}(G)=\lambda_{j, 1}\left(T_{\infty}(r)\right)$.

3. Optimality with $\frac{j}{k}>\boldsymbol{r}$. In this section we consider the structure of $(j, k, r)$ optimal graphs for $\frac{j}{k}>r$. As noted in Theorem 2.1, such graphs have $\lambda_{j, k}$-number $j+(2 r-2) k$.

THEOREM 3.1. For $\frac{j}{k}>r$, if $G$ is $(j, k, r)$-optimal, then $G$ is bipartite with $|V(G)| \equiv 0(\bmod 2 r)$.

Proof. Let $L$ be a $(j, k, r)$-optimal labeling of $G$. Since the span of $L$ is $j+(2 r-2) k$, each vertex in $V(G)$ has a label in exactly one of the three intervals $X_{1}=[0,(r-1) k]$, $X_{2}=[(r-1) k+1, j+(r-1) k-1]$, and $X_{3}=[j+(r-1) k, j+(2 r-2) k]$. Suppose $L(v) \in$ $X_{2}$, and suppose that exactly $m$ neighbors of $v$ have labels less than $L(v), 0<m<r$. Then the smallest label among the neighbors of $v$ is at most $L(v)-j-(m-1) k$, and the largest label among the neighbors of $v$ is at least $L(v)+j+(r-m-1) k$. The span of $L$ is 
thus at least $L(v)+j+(r-m-1) k-(L(v)-j-(m-1) k)=2 j+(r-2) k>j+(2 r-2) k$, a contradiction. Arguing similarly, if $m=0$, then the largest label among the neighbors of $v$ is at least $j+(2 r-2) k+1$, a contradiction. And if $m=r$, then the smallest label among the neighbors of $v$ is at most -1 , another contradiction. Hence each label assigned by $L$ is in $X_{1}$ or $X_{3}$. For $i \in\{1,3\}$, no two distinct vertices in $X_{i}$ are adjacent since the length $X_{i}$ is less than $j$. Hence, $G$ is bipartite.

Now let $v \in V(G)$ with $L(v) \in X_{1}$. Then the $r$ neighbors of $v$ have labels in $X_{3}$. Since the neighbors of $v$ are pairwise distance two apart, their labels pairwise differ by at least $k$, and hence the labels of the neighbors of $v$ must be $j+(r-1+i) k$, $0 \leq i \leq r-1$. A similar argument demonstrates that each vertex with label in $X_{3}$ has neighbors with labels $i k, 0 \leq i \leq r-1$. Thus, there are exactly $2 r$ distinct labels under $L$ with non-zero multiplicity; in $X_{1}$, these are $0, k, 2 k, \ldots,(r-1) k$, and in $X_{3}$ these are $j+(r-1) k, j+r k, \ldots, j+(2 r-2) k$.

Let $x_{1}$ and $x_{3}$ be labels assigned by $L$ in $X_{1}$ and $X_{3}$, respectively. Then we have seen that each vertex in $M_{x_{1}}$ is adjacent to some vertex in $M_{x_{3}}$. Moreover, due to the distance two condition, no two vertices in $M_{x_{1}}$ can be adjacent to the same vertex in $M_{x_{3}}$. Thus $m_{x_{1}} \leq m_{x_{3}}$. Similarly, $m_{x_{1}} \geq m_{x_{3}}$, implying $m_{x_{1}}=m_{x_{3}}$. Hence, since $L$ partitions $V(G)$ into $2 r$ nonempty labeling classes, $|V(G)|=2 r m_{x_{1}}$, from which the result follows.

We next characterize those graphs in $\Gamma(j, k, r), \frac{j}{k}>r$. It can be easily seen that $K_{r, r}$, the complete $r$-regular bipartite graph of smallest order, is the graph of smallest order in $\Gamma(j, k, r)$ (see [7]). We also point out that the converse of Theorem 3.1 is not true. For example, the graph $3 Q_{3}$, the sum of 3 copies of the 3 -cube, is a 3 -regular bipartite graph with order 24 ; however, for $\frac{j}{k}>3, \lambda_{j, k}\left(3 Q_{3}\right)=\lambda_{j, k}\left(Q_{3}\right)=j+5 k$ (see [5]). Alternatively, we observe that a graph $G$ is $(j, k, r)$-optimal if and only if each component of $G$ is $(j, k, r)$-optimal. So, since Theorem 3.1 implies that $Q_{3}$ is not $(j, k, r)$-optimal, neither is $3 Q_{3}$.

TheOREM 3.2. Let $G$ be an $r$-regular graph with $|V(G)| \equiv 0(\bmod 2 r)$. Then $G \in$ $\Gamma(j, k, r)$ if and only if there exists a partition of $V(G)$ into sets $A_{0}, A_{1}, A_{2}, \ldots, A_{r-1}$, $B_{0}, B_{1}, B_{2}, \ldots, B_{r-1}$ such that for each $i, 0 \leq i \leq r-1$, every vertex $v$ in $A_{i}$ (resp., $B_{i}$ ) has exactly one neighbor in $B_{j}$ (resp., $A_{j}$ ), $0 \leq j \leq r-1$.

Proof. $(\Rightarrow)$ Let $L$ be a $(j, k, r)$-optimal labeling of $G$. Then the result follows from the proof of Theorem 3.1 with $A_{i}$ equal to the set of vertices with label $i k$ under $L$ and $B_{i}$ equal to the set of vertices with label $j+(r-1+i) k$ under $L, 0 \leq i \leq r-1$.

$(\Leftarrow)$ The vertices in each set $A_{i}$ (resp., $B_{i}$ ) are pairwise distance three or more apart. Additionally, for $i \neq j$, each vertex in $A_{i}$ (resp., $B_{i}$ ) is distance two or more from each vertex in $A_{j}$ (resp., $B_{i}$ ). Thus, we form an $L(j, k)$-labeling $L$ of $G$ by assigning $i k$ to each vertex in $A_{i}, 0 \leq i \leq r-1$, and $j+(r-1+i) k$ to each vertex in $B_{i}, 0 \leq i \leq r-1$. Since the span of $L$ is $j+(2 r-2) k$, we are done.

DeFINITION 3.3. Let $B=X \bigcup Y$ be an $r$-regular bipartite graph and let $\mathcal{L}$ be an edge coloring of $B$ such that

(i) for each $x \in X$, the edges incident to $x$ are assigned the same color under $\mathcal{L}$,

(ii) for each $y \in Y$, the edges incident to $y$ are assigned distinct colors under $\mathcal{L}$. Then $\mathcal{L}$ is called an $X$-block coloring of $B$. We denote the minimum number of colors assigned by $X$-block colorings of $B$ by $\zeta_{X}(B)$, and if $\mathcal{L}$ is an $X$-block coloring of $B$ which assigns exactly $\zeta_{X}(B)$ distinct colors, then $\mathcal{L}$ is called a minimum $X$-block coloring of $B$.

We observe that $r \leq \zeta_{X}(B) \leq|X|$. To illustrate, we note that for either bipartition $X \bigcup Y$ of $K_{r, r}, \zeta_{X}\left(K_{r, r}\right)=r$ and for any bipartition $X \bigcup Y$ of $C_{6}, \zeta_{X}\left(C_{6}\right)=3$. 
THEOREM 3.4. Let $G$ be an r-regular bipartite graph with bipartition $W_{1}$ and $W_{2}$. Then for $\frac{j}{k}>r, G \in \Gamma(j, k, r)$ if and only if $\zeta_{W_{1}}(G)=\zeta_{W_{2}}(G)=r$.

Proof. $(\Rightarrow)$ By Theorem 3.2, we let $W_{1}=\bigcup_{i=1}^{r-1} A_{i}$ and $W_{2}=\bigcup_{i=1}^{r-1} B_{i}$. We form a $W_{1}$ (resp., $W_{2}$ )-block coloring using $r$ colors $c_{0}, c_{1}, \ldots, c_{r-1}$ by assigning color $c_{i}$ to each edge which is adjacent to some vertex in $A_{i}$ (resp., $B_{i}$ ), $0 \leq i \leq r-1$. But $\zeta_{W_{1}}(G) \geq r$ (resp., $\zeta_{W_{2}}(G) \geq r$ ) since the degree of each vertex in $B_{0}$ (resp., $A_{0}$ ) is $r$. So $\zeta_{W_{1}}(G)=r$ (resp., $\left.\zeta_{W_{2}}(G)=r\right)$.

$(\Leftarrow)$ For $i=1,2$, let $C_{i}$ be minimum $W_{i}$-block colorings of $G$. We produce a vertex labeling $L$ as follows: for each vertex $v$ in $W_{1}$ whose incident edges receive color $c_{i}$ under $C_{1}, 0 \leq i \leq r-1$, let $L(v)=i k$, and for each vertex in $W_{2}$ whose incident edges receive color $c_{i}$ under $C_{2}$, let $L(v)=j+(r-1) k+i k$. To see that $L$ is a $(j, k)$-labeling, we note that the difference between the largest label among the vertices in $W_{1}$ and the smallest label among the vertices in $W_{2}$ is $j+(r-1) k-(r-1) k=j$, implying that the labels of adjacent vertices differ by at least $j$. To show that the distance two condition is satisfied by $L$, it suffices to show that two vertices distance two apart receive different labels under $L$. If $x_{1}$ and $x_{2}$ are distance two apart with $L\left(x_{1}\right)=L\left(x_{2}\right)$ and $x_{1}, x_{2} \in W_{1}$ (resp., $W_{2}$ ), then there exists vertex $y \in W_{2}$ (resp., $W_{1}$ ) and edges $\left\{x_{1}, y\right\}$ and $\left\{x_{2}, y\right\}$ which receive the same color under $C_{1}$ (resp., $C_{2}$ ), a contradiction.

We illustrate a 3-regular bipartite graph $B=X \bigcup Y$ on 12 vertices with $\zeta_{X}(B)=$ 3 and $\zeta_{Y}(B)=5$. For $X=\left\{x_{1}, x_{2}, x_{3}, x_{4}, x_{5}, x_{6}\right\}$ and $Y=\left\{y_{1}, y_{2}, y_{3}, y_{4}, y_{5}, y_{6}\right\}$, let the neighborhood set of $x_{i}$, denoted $N\left(x_{i}\right)$, be as follows:

$$
\begin{aligned}
& N\left(x_{1}\right)=\left\{y_{1}, y_{2}, y_{3}\right\}, \\
& N\left(x_{2}\right)=\left\{y_{4}, y_{5}, y_{6}\right\}, \\
& N\left(x_{3}\right)=\left\{y_{1}, y_{3}, y_{5}\right\}, \\
& N\left(x_{4}\right)=\left\{y_{2}, y_{4}, y_{6}\right\}, \\
& N\left(x_{5}\right)=\left\{y_{1}, y_{3}, y_{4}\right\}, \\
& N\left(x_{6}\right)=\left\{y_{2}, y_{5}, y_{6}\right\} .
\end{aligned}
$$

Then for $1 \leq i \leq 3$, assigning color $i$ to the edges incident to $x_{2 i-1}$ and $x_{2 i}$ shows that $\zeta_{X}(B)=3$. On the other hand, examination of the neighborhood sets of each $y_{i}$ gives $\zeta_{Y}(B)=5$.

THEOREM 3.5. For $r \geq 3$, let $j, k, j^{\prime}$, and $k^{\prime}$ be integers such that $\frac{j}{k}>r$ and $\frac{j^{\prime}}{k^{\prime}}>r$. Then $\Gamma(j, k, r)=\Gamma\left(j^{\prime}, k^{\prime}, r\right)$.

Proof. Let $G \in \Gamma(j, k, r)$. By Theorem 3.1, $G$ is bipartite, so $G$ can be expressed $X \bigcup Y$. This implies $\zeta_{X}(G)=\zeta_{Y}(G)=r$ by Theorem 3.4, which in turn implies (also by Theorem 3.4) that $G \in \Gamma\left(j^{\prime}, k^{\prime}, r\right)$. Thus $\Gamma(j, k, r) \subseteq \Gamma\left(j^{\prime}, k^{\prime}, r\right)$. A similar argument shows $\Gamma\left(j^{\prime}, k^{\prime}, r\right) \subseteq \Gamma(j, k, r)$.

Let $x=\frac{j}{k}$. In [4], it is shown that for any graph $G$, the function $\lambda_{x}(G)=\frac{1}{k} \lambda_{j, k}(G)$ is continuous in $x$ on the set of rationals greater than or equal to 1 . (Here, continuity at rational number $x \geq 1$ means for any real $\epsilon>0$, there exists real $\delta>0$ such that for rational $q \geq 1$ within $\delta$ of $x, \lambda_{x}(G)$ is within $\epsilon$ of $\lambda_{q}(G)$.) Additionally, we have seen that, if $H \in \Gamma(j, k, r), \frac{j}{k}>r$, then $\lambda_{x}(H)=x+(2 r-2)$. Thus $\lambda_{r}(H)=r+(2 r-2)$, which establishes that $H \in \Gamma(a r, a, r)$ for $a \in Z^{+}$. It follows that for $\frac{j}{k}>r, \Gamma(j, k, r) \subseteq \Gamma(a r, a, r)$. We point out, however, that for $\frac{j}{k}>r, \Gamma(j, k, r)$ and $\Gamma(a r, a, r)$ are not equal. As an example, $K_{3}$, which is not bipartite and hence not optimal for $\frac{j}{k}>2$, is a member of $\Gamma(2,1,2)$. 
4. Optimality with $\frac{j}{k} \in Z^{+}$and $\frac{j}{k}<r$. In this section, we investigate the structure of $\Gamma(j, k, r)$ for $\frac{j}{k}$ an integer. Since $\lambda_{j, k}(G)=k \lambda_{c, 1}(G)$ for $\frac{j}{k}=c \in Z^{+}$, it will suffice to assume $k=1$.

We begin with a consideration of $\Gamma(r-1,1, r)$ for $r \geq 2$.

TheOREM 4.1. For $r \geq 2, \Gamma(r-1,1, r) \subseteq \Gamma(r, 1, r)$.

Proof. If $G \in \Gamma(r-1,1, r)$, then $\lambda_{r-1,1}(G)=3 r-4$ by Theorem 2.2. Let $L$ be a $\lambda_{r-1,1}$-labeling of $G$. Then $L^{\prime}(x)=L(x)+\left\lfloor\frac{L(x)}{r-1}\right\rfloor$ is an $L(r, 1)$-labeling of $G$ with span $3 r-2$.

It follows from the discussion at the end of section 3 that for all $a \in Z^{+}$and $\frac{j^{\prime}}{k^{\prime}}>r, \Gamma(a(r-1), a, r) \bigcup \Gamma\left(j^{\prime}, k^{\prime}, r\right) \subseteq \Gamma(a r, a, r)$. We next turn our attention to a special class of optimal labelings.

DeFINITION 4.2. Let $1 \leq j \leq r$ and let $L$ be a $(j, 1, r)$-optimal labeling of $r$-regular graph $G$. Then $L$ is said to be a $(j, 1, r)$-cyclically optimal labeling of $G$ if and only if for any adjacent vertices $v_{i}$ and $v_{i^{\prime}}$ in $V(G), L\left(v_{i}\right) \notin\left\{L\left(v_{i^{\prime}}\right) \pm j^{\prime}\left(\bmod \lambda_{j, 1}(G)+\right.\right.$ 1) $\left.\mid 0 \leq j^{\prime} \leq j-1\right\}$. If $G$ has a $(j, 1, r)$-cyclically optimal labeling, then $G$ is said to be $(j, 1, r)$-cyclically optimal; otherwise, $G$ is $(j, 1, r)$-acyclically optimal. We denote the collection of $(j, 1, r)$-cyclically optimal graphs by $\Gamma_{c}(j, 1, r)$.

To illustrate, we give a $(3,1,4)$-cyclically optimal labeling of a graph in Figure 4.1. We also point out that $K_{3}$ is an element of $\Gamma(2,1,2)$ but not of $\Gamma_{c}(2,1,2)$.

We also note that $\Gamma_{c}(1,1, r)$ necessarily equals $\Gamma(1,1, r)$.

THEOREM 4.3. Let $G$ be a $(j, 1, r)$-cyclically optimal graph, where $j \leq r$. Then $|V(G)| \equiv 0(\bmod r+2 j-1)$.

Proof. Let $L$ be a $(j, 1, r)$-cyclically optimal labeling of graph $G$ with span $2 j+r-2$ by Theorem 2.2. It suffices to show $m_{0}=m_{1}=\cdots=m_{r+2 j-2}$.

By the definition of cyclic labeling, the $r$ neighbors of any vertex $v$ with label $L(v)=x$ must have labels which are precisely the elements of $S_{x}=\{(L(v)+j+$ i) $(\bmod r+2 j-1) \mid 0 \leq i \leq r-1\}$. Thus, since $v$ cannot be adjacent to two vertices with the same label, we have $m_{x} \leq m_{y}$ for every $y$ in $S_{x}$. But if $y \in S_{x}$, then $x \in S_{y}$, so $m_{y} \leq m_{x}$. Thus $m_{i}=m_{j+i}=m_{j+i+1}=m_{i+1}$ for $0 \leq i \leq j+r-3$, giving the result.

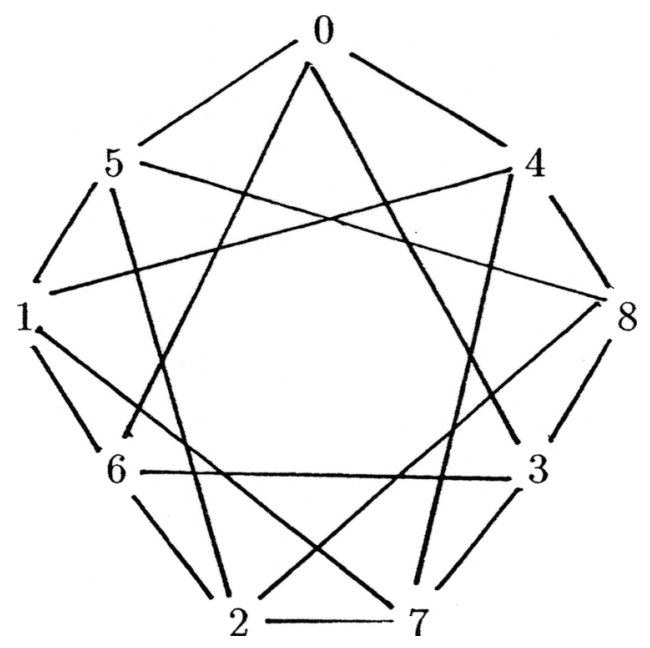

FIG. 4.1. A $(3,1,4)$-cyclically optimal labeling of graph $G$. 
Corollary 4.4. If $G \in \Gamma(1,1, r)$, then $|V(G)| \equiv 0(\bmod r+1)$.

Proof. If $G \in \Gamma(1,1, r)$, then $G$ is necessarily $(1,1, r)$-cyclically optimal. The result follows immediately from Theorem 3.1.

We note that the converse to Corollary 4.4 is not true since the $\lambda_{1,1}$-number of $C_{4}+C_{5}$ (the sum of $C_{4}$ and $C_{5}$ ) is 4 .

Theorem 4.5. Let $r \geq 2$. If for fixed $j, 1 \leq j \leq r, G$ is bipartite and $(j, 1, r)$ cyclically optimal, then $|V(G)| \equiv 0(\bmod 2 r+4 j-2)$.

Proof. Let $L$ be a $(j, 1, r)$-cyclically optimal labeling of the bipartite graph $G=$ $X \cup Y$. As in the proof of Theorem 3.1, it can be easily shown that each of the $r+2 j-1$ labels has the same multiplicity. Since $L$ is cyclic, the subgraph of $G$ induced by $M_{i} \bigcup M_{i+j} \bigcup M_{i+2 j}, 0 \leq i \leq r-2$, is 2-regular and thus is a sum of even cycles each of which has order divisible by 6 . It follows that $\left|M_{i} \bigcap X\right|=\left|M_{i} \cap Y\right|$. Hence, each $m_{i}$ is even, which establishes the theorem.

We now give a constructive characterization of $\Gamma_{c}(j, 1, r)$. Let $n$ and $h$ be fixed, $n \geq 3$ and $1 \leq h \leq\left\lfloor\frac{n}{2}\right\rfloor$. Then the generalized $h$-cycle on $n$ vertices, denoted ${ }_{h} C_{n}$, is the graph with vertex set $\left\{v_{0}, v_{1}, v_{2}, \ldots, v_{n-1}\right\}$ and edge set $\left\{v_{i} v_{s} \mid 0 \leq i \leq n-1\right.$ and $s=(i+l)(\bmod n), 1 \leq l \leq h\}$. We note that ${ }_{h} C_{n}$ is isomorphic to $C_{n}$ and $K_{n}$ when, respectively, $h=1$ and $h=\left\lfloor\frac{n}{2}\right\rfloor$.

Now fix $r, j$, and $m, j \leq r$. For $1 \leq i \leq m$, let $G_{i}$ be the graph on $r+2 j-1$ vertices $v_{i, 0}, v_{i, 1}, v_{i, 2}, \ldots, v_{i, r+2 j-2}$ such that for all $l, v_{i, l}$ is adjacent to precisely every vertex in $V\left(G_{i}\right)$ except $v_{i, l \pm x(\bmod r+2 j-1)}, 0 \leq x \leq j-1$. (We note that $G_{i}$ is isomorphic to $\overline{{ }_{h} C_{n}}$, where $h=j-1$ and $n=r+2 j-1$.) Then it is easily verified that $G_{i}$ is in $\Gamma_{c}(j, 1, r)$ and that the labeling $L_{i}$ of $G_{i}$ such that $L_{i}\left(v_{i, x}\right)=x$ is a $(j, 1, r)$-cyclically optimal labeling. Consequently, the graph $G=\sum_{i=1}^{m} G_{i}$ is a $(j, 1, r)$-cyclically optimal graph and the labeling of $G$ given by $L\left(v_{i, x}\right)=x$ is a $(j, 1, r)$-cyclically optimal labeling.

Let $\mathcal{M}_{0}$ be the singleton set containing $G$, and let $\mathcal{M}_{1}, \mathcal{M}_{2}, \mathcal{M}_{3} \ldots$ be defined recursively as follows: for $y \geq 1, G^{\prime \prime} \in \mathcal{M}_{y}$ if and only if for some graph $G^{\prime} \in \mathcal{M}_{y-1}$ with edges $v_{i_{1}, x_{1}} v_{i_{1}, x_{2}}$ and $v_{i_{3}, x_{1}} v_{i_{2}, x_{2}}, G^{\prime \prime}$ results from the following edge transpositions on $G^{\prime}$ :

1. Delete $v_{i_{1}, x_{1}} v_{i_{1}, x_{2}}$.

2. Delete $v_{i_{3}, x_{1}} v_{i_{2}, x_{2}}$.

3. Add $v_{i_{1}, x_{1}} v_{i_{2}, x_{2}}$.

4. Add $v_{i_{1}, x_{2}} v_{i_{3}, x_{1}}$.

Then by induction, each graph $G$ in $\bigcup_{y=0} \mathcal{M}_{y}$ is $r$-regular with cyclic labeling $L$, since the effect of the edge transpositions is to redirect two edges from vertices labeled $x_{1}$ and $x_{2}$ to vertices with labels $x_{1}$ and $x_{2}$. Thus $\bigcup_{y=0} \mathcal{M}_{y} \subseteq \Gamma_{c}(j, 1, r)$.

To show that $\Gamma_{c}(j, 1, r) \subseteq \bigcup_{y=0} \mathcal{M}_{y}$, let $G$ be a $(j, 1, r)$-cyclically optimal graph and let $L$ be a $(j, 1, r)$-cyclically optimal labeling of $G$. Then Theorem 4.3 implies that $|V(G)|=c(r+2 j-1)$ for some $c$ and that we may thus denote the vertices of $G$ by $v_{i, z}, 1 \leq i \leq c$ and $0 \leq z \leq r+2 j-2$, where $L\left(v_{i, z}\right)=z$. Furthermore, since the $r$ neighbors of $v_{i, x}$ necessarily have labels $(x+y)(\bmod r+2 j-1), j \leq y \leq r+j-1$, it is the case that for every $i_{1}, i_{2}, x_{1}, x_{2}$ such that $i_{1} \neq i_{2}$ and $v_{i_{1}, x_{1}}$ is adjacent to $v_{i_{2}, x_{2}}$, there exists $i_{3} \neq i_{1}$ such that $v_{i_{1}, x_{2}}$ is adjacent to $v_{i_{3}, x_{1}}$. Hence, for $i_{1} \neq i_{2}$, an $r$-regular graph $G^{\prime}$ may be formed by executing the following algorithm, which may be thought of as a reversal of the edge manipulation algorithm given above:

a. Delete $v_{i_{1}, x_{1}} v_{i_{2}, x_{2}}$.

b. Delete $v_{i_{1}, x_{2}} v_{i_{3}, x_{1}}$.

c. Add $v_{i_{1}, x_{1}} v_{i_{1}, x_{2}}$.

d. Add $v_{i_{3}, x_{1}} v_{i_{2}, x_{2}}$. 
Moreover, the vertex labeling $L^{\prime}\left(v_{i, x}\right)$ of $G^{\prime}$ given by $L^{\prime}=L$ is a $(j, 1, r)$-optimal labeling, since the effect of these edge manipulations is to redirect two edges from vertices labeled $x_{1}$ and $x_{2}$ to vertices labeled $x_{1}$ and $x_{2}$.

As compared to $G$, the graph $G^{\prime}$ produced by this algorithm has 1 (or 2) fewer edges of the form $v_{a, x_{i}} v_{b, x_{h}}$ where $a \neq b$, and 1 (or 2) more edges of the form $v_{c, x_{i}} v_{c, x_{h}}$. The algorithm may thus be iterated sufficiently many times to produce $\sum_{i=1}^{m} G_{i}$, each of whose edges is of the form $v_{c, x_{i}} v_{c, x_{h}}$. Hence, $\Gamma_{c}(j, 1, r) \subseteq \bigcup_{y=0} \mathcal{M}_{y}$, which in turn implies the following.

THEOREM 4.6. Every $(j, 1, r)$-cyclically optimal graph yields, through a sequence of edge transpositions, a graph isomorphic to a sum of copies of $\overline{j-1} C_{r+2 j-1}$.

From this construction, we have the following.

COROLlary 4.7. A connected graph $G$ is $(j, 1, r)$-cyclically optimal if and only if there exists a partition $\left\{V_{0}, V_{1}, V_{2}, \ldots, V_{r+2 j-2}\right\}$ of $V(G)$ such that, for $0 \leq i \leq$ $r+2 j-2$, each vertex in $V_{i}$ is adjacent to exactly one vertex in $V_{\left(i+i^{\prime}\right)(\bmod r+2 j-1)}$, $j \leq i^{\prime} \leq j+r-1$. Necessarily, the sets in the partition are of equal size.

5. Optimality with $\frac{j}{k}=2$. In this section, we investigate the graphs in $\Gamma(2,1, r), r \geq 2$, each of which has $\lambda_{2,1}$-number equal to $r+2$. Since, in general, not all $(2,1, r)$-optimal labelings of $r$-regular graphs are cyclic, then the $r+3$ labels given by an optimal labeling $L$ need not have equal multiplicities. However, as we shall see, the multiplicities of labels under a $(2,1, r)$-optimal labeling $L$ do possess certain regularities.

We note that for $r=2$, any graph in $\Gamma(2,1, r)$ is a cycle $C_{n}$. Moreover, since $\lambda_{2,1}\left(C_{n}\right)=4=r+2$, it follows that $\Gamma(2,1,2)=\left\{C_{n} \mid n \geq 3\right\}$. It thus suffices to consider $r \geq 3$.

Theorem 5.1. Let $G$ be a $(2,1, r)$-optimal graph, where $r \geq 3$, and let $L$ be a $\lambda_{2,1}$-labeling of $G$. Then $m_{i}=m_{h}$ for $1 \leq i, h \leq r+1$.

Proof. Let $T$ be the set of integers in the interval [0, $r+2]$. Then for every integer $x, 1 \leq x \leq r+1$, there are exactly $r$ elements in $T$ which differ from $x$ by at least 2. Thus, the distance conditions require the labels of the neighbors of each vertex $v$ with label $L(v)=x$ to be precisely the elements of $S_{x}=\{w \in T|| x-w \mid \geq 2\}$. So, for every $y \in S_{x}$, we have $m_{x} \leq m_{y}$. But if $y \in S_{x}$ where $1 \leq y \leq r+1$, then $x \in S_{y}$, implying $m_{y} \leq m_{x}$. Hence $m_{h}=m_{2+h}=m_{3+h}=m_{h+1}$ for $1 \leq h \leq r-2$, giving the result.

Now let $L$ be a $(2,1, r)$-optimal labeling of $r$-regular graph $G$. We define $M(\alpha, \beta)$ to be the set of vertices in $V(G)$ which have label $\alpha$ and which are adjacent to some vertex with label $\beta$, and we denote the cardinality of $M(\alpha, \beta)$ by $m(\alpha, \beta)$. Noting that, for $v$ such that $L(v)=0$, exactly one element $i$ of the set $\{2,3,4, \ldots, r+2\}$ is not represented among the labels of the neighbors of $v$, we define $M\left(0, i^{*}\right)$ to be the collection of vertices which are labeled 0 and which are adjacent to no vertices labeled $i$. For $i \in\{0,1,2,3,4, \ldots, r\}$, we define $M\left(r+2, i^{*}\right)$ analogously, and we denote the cardinalities of $M\left(0, i^{*}\right)$ and $M\left(r+2, i^{*}\right)$ by $m\left(0, i^{*}\right)$ and $m\left(r+2, i^{*}\right)$, respectively. For fixed $h, 2 \leq h \leq r+1$, there is a one-to-one correspondence between $M_{h}$ and $M_{0}-M\left(0, h^{*}\right)$, implying that $m_{h}=m_{0}-m\left(0, h^{*}\right)$. So, by Theorem 5.1, it follows that $m\left(0,2^{*}\right)=m\left(0,3^{*}\right)=\cdots=m\left(0, r+1^{*}\right)$. Similarly, for $1 \leq i \leq r$, $m\left(r+2,1^{*}\right)=m\left(r+2,2^{*}\right)=\cdots=m\left(r+2, r^{*}\right)$.

Since $m(0, r+2)=m(r+2,0)$, we have

$$
\sum_{i=2}^{r+1} m\left(0, i^{*}\right)=m(0, r+2)=m(r+2,0)=\sum_{i=1}^{r} m\left(r+2, i^{*}\right) .
$$


But for every $i, 2 \leq i \leq r+1$, each vertex in $M_{i}$ is adjacent to some vertex in $M_{0}$, implying $m(0, i)=m_{i}$. Thus, since $m(i, 0)=m(0, i)$, we have $m_{0}=m(0, i)+$ $m\left(0, i^{*}\right)=m_{i}+m\left(0, i^{*}\right)$, giving $m_{0}-m_{i}=m\left(0, i^{*}\right)$. But $m_{2}=m_{3}=\cdots=m_{r+1}$ by Theorem 5.1, so $m\left(0, i^{*}\right)=m_{0}-m_{2}$ for every $i, 2 \leq i \leq r+1$.

Similarly, $m\left(r+2, i^{*}\right)=m_{r+2}-m_{2}$, which, by (1), implies

$$
\sum_{i=2}^{r+1}\left(m_{0}-m_{2}\right)=\sum_{i=1}^{r}\left(m_{r+2}-m_{2}\right) .
$$

This gives the following theorem.

Theorem 5.2. Let $G \in \Gamma(2,1, r)$ and let $L$ be a $\lambda_{2,1}$-labeling of $G$. Then $m_{0}=$ $m_{r+2}$.

For fixed $h, 2 \leq h \leq r+1$, there is a one-to-one correspondence between $M_{h}$ and $M_{0}-M\left(0, h^{*}\right)$, implying that $m_{h}=m_{0}-m\left(0, h^{*}\right)$ (and likewise, $m_{h}=m_{r+2}-m(r+$ $\left.2, h^{*}\right)$ for $\left.1 \leq h \leq r\right)$. So, by Theorems 5.1, 5.2, and (1), it follows that

$$
\begin{gathered}
m\left(0,2^{*}\right)=m\left(0,3^{*}\right)=\cdots=m\left(0, r+1^{*}\right) \\
=m\left(r+2,1^{*}\right)=m\left(r+2,2^{*}\right)=\cdots=m\left(r+2, r^{*}\right) .
\end{gathered}
$$

We use this result to establish the next theorem.

TheOrem 5.3. Let $G \in \Gamma(2,1, r)$ and let $L$ be a $\lambda_{2,1}$-labeling of $G$. Then $|V(G)|=$ $(r+3) m\left(0, r+2^{*}\right)+\left(r^{2}+2 r-1\right) m\left(0,2^{*}\right)$.

Proof. By Theorems 5.1 and 5.2, $|V(G)|=\sum_{i=0}^{r+2} m_{i}=2 m_{0}+(r+1) m_{2}$. Since

$$
m_{0}=\sum_{i=2}^{r+2} m\left(0, i^{*}\right)=r m\left(0,2^{*}\right)+m\left(0, r+2^{*}\right)
$$

and

$$
\begin{aligned}
m_{2} & =m_{0}-m\left(0,2^{*}\right)=-m\left(0,2^{*}\right)+\sum_{i=2}^{r+2} m\left(0, i^{*}\right)=\sum_{i=3}^{r+2} m\left(0, i^{*}\right) \\
& =m\left(0, r+2^{*}\right)+\sum_{i=3}^{r+1} m\left(0, i^{*}\right)=m(0, r+2)^{*}+(r-1) m\left(0,2^{*}\right),
\end{aligned}
$$

the result now follows via straightforward algebra.

Since $m\left(0,2^{*}\right)$ and $m\left(0, r+2^{*}\right)$ must be nonnegative, we observe that the smallest $(2,1, r)$-optimal graph has order at least $r+3$. As noted in the preceding section, this bound is achieved by the unique $r$-regular graph on $r+3$ vertices: $\overline{C_{r+3}}$, which is cyclically optimal. If $G \in \Gamma(2,1, r)$ is acyclically optimal, then every optimal labeling of $G$ has $m(0, r+2) \geq 1$, which in turn implies that $m\left(0,2^{*}\right) \geq 1$. Thus, the smallest $(2,1, r)$-acyclically optimal graph has order at least $r^{2}+2 r-1$. We produce a $(2,1, r)$-acyclically optimal graph $G_{a}(r)$ on $r^{2}+2 r-1$ vertices as follows: noting that $m_{0}=m_{r+2}=r$ and $m_{1}=m_{2}=\cdots=m_{r+1}=r-1$ under an acyclically optimal labeling of $G$, we define

$$
\begin{aligned}
& M_{i}=\left\{v_{i}^{h}, 1 \leq h \leq r-1\right\} \text { for } 1 \leq i \leq r+1, \text { and } \\
& M_{i}=\left\{v_{i}^{p}, 1 \leq p \leq r\right\} \text { for } i=0, r+2 .
\end{aligned}
$$

Let $P_{0}=\left\{S_{1}, S_{2}, S_{3}, \ldots, S_{r}\right\}$, where $S_{i}$ is lexicographically the $i$ th subset size $r-1$ of $\{2,3,4, \ldots, r+1\}$. Similarly, let $P_{r+2}=\left\{T_{1}, T_{2}, T_{3}, \ldots, T_{r}\right\}$, where $T_{i}$ is lexico- 


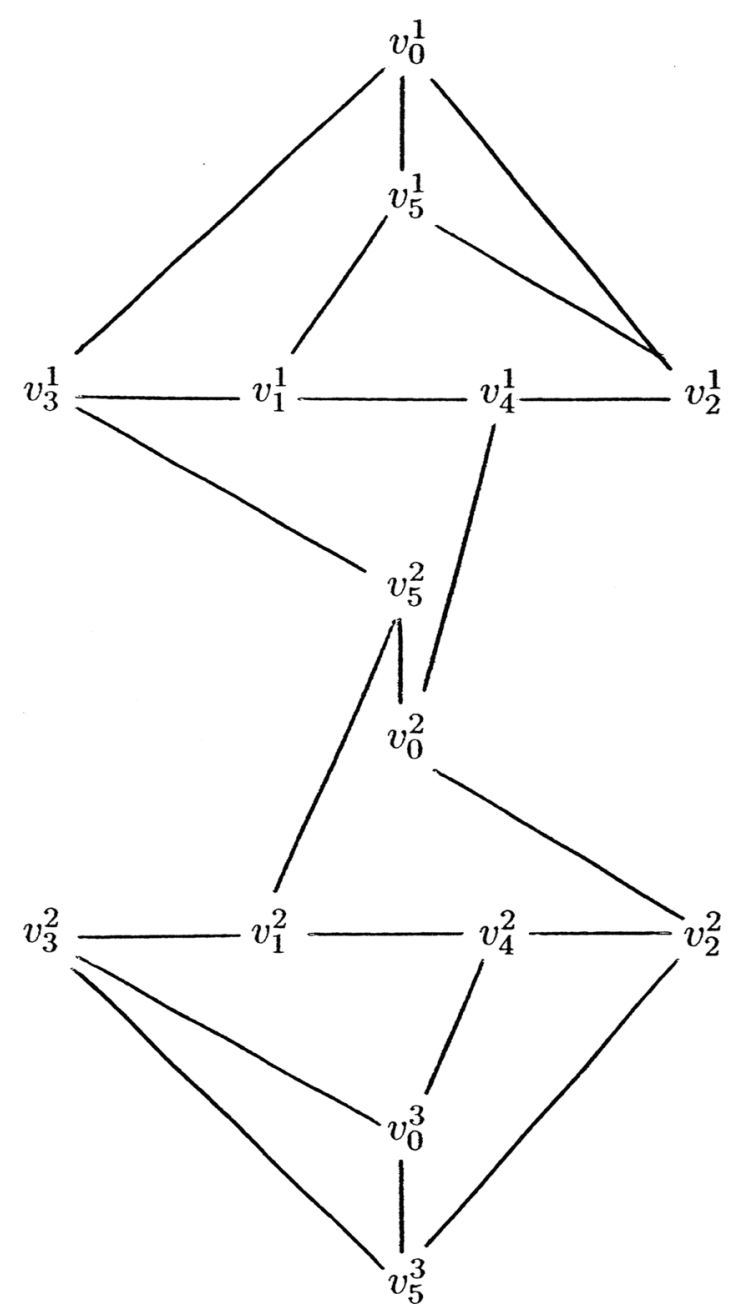

FIG. 5.1. A $\lambda_{2,1}$-labeling of a $(2,1,3)$-acyclically optimal graph on 14 vertices.

graphically the $i$ th subset size $r-1$ of $\{1,2,3, \ldots, r\}$. We define the edges of $G$ as follows:

1. For $1 \leq p \leq r,\left\{v_{0}^{p}, v_{r+2}^{p}\right\} \in E(G)$.

2. For $1 \leq h \leq r-1,\left\{v_{s}^{h}, v_{t}^{h}\right\} \in E(G)$ if and only if $|s-t| \geq 2,1 \leq s, t \leq r+1$.

3. For $1 \leq p \leq r, 2 \leq i \leq r+1$ and $1 \leq h \leq r-1,\left\{v_{0}^{p}, v_{i}^{h}\right\} \in E(G)$ if and only if $S_{p}$ contains $i$ and there are exactly $h-1$ sets $S_{1}, S_{2}, \ldots, S_{p-1}$ which contain $i$.

4. For $1 \leq p \leq r, 1 \leq i \leq r$ and $1 \leq h \leq r-1,\left\{v_{r+2}^{p}, v_{i}^{h}\right\} \in E(G)$ if and only if $T_{p}$ contains $i$ and there are exactly $h-1$ sets $T_{1}, T_{2}, \ldots, T_{p-1}$ which contain $i$.

Then the labeling $L$ given by $L\left(v_{z}^{i}\right)=z$ is a $(2,1, r)$-acyclically optimal labeling of $G$. In Figure 5.1, we illustrate a $\lambda_{2,1}$-labeling of a $(2,1,3)$-acyclically optimal graph on 14 vertices.

The existence of $(2,1, r)$-optimal graphs on $r+3$ vertices and on $r^{2}+2 r-1$ vertices leads to the following theorem. 
TheOrem 5.4. For $x, y \in Z^{+}$, there exists a $(2,1, r)$-optimal graph on $x(r+3)+$ $y\left(r^{2}+2 r-1\right)$ vertices.

COROLlaRY 5.5. If $r$ is even, then for all $n \geq(r+2)\left(r^{2}+2 r-2\right)$, there exists a $(2,1, r)$-optimal graph on $n$ vertices. If $r$ is odd, then for all $n \geq \frac{(r-5)\left(r^{2}+2 r-3\right)}{4}$, there exists a $(2,1, r)$-optimal graph on $2 n$ vertices.

Proof. If $r$ is even, then $\operatorname{gcd}\left(r+3, r^{2}+2 r-1\right)=1$, implying every integer greater than or equal to $(r+2)\left(r^{2}+2 r-2\right)$ can be written as a linear combination of $r+3$ and $r^{2}+2 r-1$ with nonnegative coefficients. If $r$ is odd, then $\operatorname{gcd}\left(r+3, r^{2}+2 r-1\right)=2$, giving the result by a similar argument.

Although the $(2,1, r)$-cyclically optimal graph on $r+3$ vertices is unique, such is not the case for $(2,1, r)$-acyclically optimal graphs on $r^{2}+2 r-1$ vertices. However, each $(2,1, r)$-acyclically optimal graph on $r^{2}+2 r-1$ vertices, through a sequence of edge transpositions similar to that described in the preceding section, yields a graph isomorphic to $G_{a}(r)$. Extending this argument gives the following theorem.

THEOREM 5.6. Every $(2,1, r)$-optimal graph yields, through a sequence of edge transpositions, a graph isomorphic to a sum of copies of $\overline{C_{r+3}}$ and $G_{a}(r)$.

6. On prisms. In this section, we apply our results on optimality to a special class of 3-regular graphs known as prisms. For $n \geq 3$, the $n$-prism, denoted $\operatorname{Pr}(n)$, is the graph consisting precisely of two disjoint $n$-cycles $v_{0}, v_{1}, \ldots, v_{n-1}$ and $w_{0}, w_{1}, \ldots, w_{n-1}$ and edges $\left\{v_{i}, w_{i}\right\}$ for $0 \leq i \leq n-1$. The two cycles shall be called the inner and outer cycles, respectively. We point out that $\operatorname{Pr}(n)$ is isomorphic to $C_{n} \times P_{2}$. We also note that it will be convenient to exhibit a labeling of $\operatorname{Pr}(n)$ in the form of a $2 \times n$ array, where the entries in the top row of the array correspond to the labels of the vertices of the outer cycle and the entries in the bottom row correspond to the labels of the vertices of the inner cycle.

In [14], Jha et al. proved the following theorem.

Theorem 6.1. Let $n \geq 3$. Then

$$
\lambda_{2,1}(\operatorname{Pr}(n))\left\{\begin{array}{l}
=5 \text { if } n \equiv 0(\bmod 3) \\
\leq 6 \text { if } n \neq \equiv 0(\bmod 3) .
\end{array}\right.
$$

We refine this theorem as follows (and are informed that an alternative proof will appear in [15]).

Theorem 6.2. Let $n \geq 3$. Then

$$
\lambda_{2,1}(\operatorname{Pr}(n))=\left\{\begin{array}{l}
5 \text { if } n \equiv 0(\bmod 3) \\
6 \text { if } n \neq \equiv 0(\bmod 3)
\end{array}\right.
$$

Proof. By Theorem 6.1, it suffices to show that $\lambda_{2,1}(\operatorname{Pr}(n))>5$ for $n \not \equiv 0(\bmod$ $3)$. Suppose to the contrary that there exists an $n, n \equiv 1,2(\bmod 3)$, such that $\lambda_{2,1}(\operatorname{Pr}(n))=5$. Let $L$ be a $\lambda_{2,1}$-labeling of $\operatorname{Pr}(n)$. Since the order of $\operatorname{Pr}(n)$ is $2 n$, we observe that $|V(\operatorname{Pr}(n))| \equiv 2,4(\bmod 6)$, which by Theorem 4.3 implies that $L$ is acyclic. Thus, by the discussion following Theorem $5.1, m\left(0, i^{*}\right) \geq 1,2 \leq i \leq 4$, implying $m\left(0,3^{*}\right) \geq 1$.

With no loss of generality, let $a_{0}, a_{1}, a_{2}, b_{0}, b_{1}, b_{2}$ be vertices in $V(\operatorname{Pr}(n))$ such that $a_{1} \in M\left(0,3^{*}\right)$. Then the neighbors of $a_{1}$, namely, $a_{0}, a_{2}$, and $b_{1}$, receive the labels 2 , 4 , and 5 under $L$ (not necessarily respectively). If $a_{0}$ or $b_{1}$ receives the label 2 , then by virtue of the 4 -cycle $\left\langle a_{0}, a_{1}, b_{1}, b_{0}\right\rangle, L\left(b_{0}\right) \geq 6$, contradicting the optimality of $L$. If $a_{2}$ receives the label 2 , then by virtue of the 4 -cycle $\left\langle a_{1}, a_{2}, b_{2}, b_{1}\right\rangle, L\left(b_{2}\right) \geq 6$, another contradiction. 
For $\frac{j}{k}>r$, if $\operatorname{Pr}(n) \in \Gamma(j, k, 3)$, then by Theorem 3.1, $\operatorname{Pr}(n)$ is bipartite (implying that $n$ is even) and $2 n \equiv 0(\bmod 6)$. Hence $n \equiv 0(\bmod 6)$, a condition which is easily seen to be sufficient for optimality by labeling the vertices of the inner cycle $0,2,4,0,2,4, \ldots, 0,2,4$ and the vertices of the outer cycle $3,5,1,3,5,1, \ldots, 3,5,1$.

If $\operatorname{Pr}(n) \in \Gamma(1,1,3)$, then by Theorem $4.3, \mid V(\operatorname{Pr}(n) \mid \equiv 0(\bmod 4)$. Hence, $n$ is even, so $\operatorname{Pr}(n)$ is bipartite. By Theorem $4.5, \mid V(\operatorname{Pr}(n) \mid \equiv 0(\bmod 8)$, implying the necessary condition $n \equiv 0(\bmod 4)$. However, this condition is also sufficient for the $(1,1,3)$-optimality of $\operatorname{Pr}(n)$, as shown in the definitive calculation of $\lambda_{1,1}(\operatorname{Pr}(n))$, given below.

Theorem 6.3. Let $n \geq 3$. Then

$$
\lambda_{1,1}(\operatorname{Pr}(n))=\left\{\begin{array}{l}
3 \text { if } n \equiv 0(\bmod 4) \\
5 \text { if } n=3,6 \\
4 \text { otherwise. }
\end{array}\right.
$$

Proof. For $n \equiv 0(\bmod 4)$, consider the array $A_{1}$, which represents a $\lambda_{1,1}$-labeling of $\operatorname{Pr}(4)$ :

0123

2301.

Then if $n=4 m$, an optimal $(1,1,3)$-labeling of $\operatorname{Pr}(n)$ is demonstrable by catenating $m$ copies of $A_{1}$ like so:

$$
\begin{array}{lllllllllllll}
0 & 1 & 2 & 3 & 0 & 1 & 2 & 3 & \ldots & 0 & 1 & 2 & 3 \\
2 & 3 & 0 & 1 & 2 & 3 & 0 & 1 & \ldots & 2 & 3 & 0 & 1 .
\end{array} \text {. }
$$

For $n=3,6$, consider $n=3$. Then $\operatorname{Pr}(3)$ has diameter two, which implies (by the distance conditions) that no two vertices may be assigned the same label. It is then an easy matter to show the existence of an $L(1,1)$-labeling of $\operatorname{Pr}(3)$ with span equal to the lower bound 5 . If $n=6$, then the converse of part $a$ implies that $\lambda_{1,1}(\operatorname{Pr}(6)) \geq 4$. But if $\lambda_{1,1}(\operatorname{Pr}(6))=4$, the pigeon-hole principle implies the existence of a label with multiplicity 3. The distance constraints, however, imply that no label may have multiplicity 3 . Thus, $\lambda_{1,1}(\operatorname{Pr}(6))=5$, as demonstrated by the following labeling:

\section{5}

234501 .

In the final case, we note that $n$ is not a multiple of 4 , implying that $\lambda_{1,1}(\operatorname{Pr}(n)) \geq$ 4. It thus suffices to show the existence of an $L(1,1)$-labeling with span 4 . To that end, consider the array $A_{2}$, which represents a $\lambda_{1,1}$-labeling of $\operatorname{Pr}(5)$ :

01234

$$
23401 \text {. }
$$

Then, since any integer $n, n>11$ and $n$ not divisible by 4 , can be written $4 \alpha+5 \beta$ for some $\alpha \geq 0$ and $\beta \geq 1$, we can demonstrate an $L(1,1)$-labeling with span 4 by the catenating $\alpha$ copies of $A_{1}$ and $\beta$ copies of $A_{2}$.

In the remaining cases $n=7$ and $n=11$, we demonstrate $L(1,1)$-labelings with $\operatorname{span} 4$ : 
and

$$
\begin{array}{lllllllllll}
0 & 2 & 3 & 1 & 0 & 2 & 4 & 1 & 0 & 3 & 4 \\
3 & 1 & 0 & 4 & 3 & 1 & 0 & 2 & 4 & 1 & 2 .
\end{array} .
$$

Let the Cartesian product of the infinite path $P_{\infty}$ and $P_{2}$ be denoted by $\operatorname{Pr}(\infty)$. By an approach similar to the one used in the proof of Theorem 2.3, we may establish that $\lambda_{j, k}(\operatorname{Pr}(\infty)) \leq \lambda_{j, k}(\operatorname{Pr}(n))$ for all $n \geq 3$; furthermore, it can be shown that $\lambda_{1,1}(\operatorname{Pr}(\infty))=3$ and $\lambda_{2,1}(\operatorname{Pr}(\infty))=5$ and that $\operatorname{Pr}(\infty)$ is both $(1,1,3)$ - and $(2,1,3)$ cyclically optimal. (By Theorem 4.1, $\operatorname{Pr}(\infty)$ is $(3,1,3)$-optimal.) Finally, analysis analogous to that employed in the proof of Theorem 6.2 reveals that all optimal $(2,1,3)$-labelings of $\operatorname{Pr}(\infty)$ are cyclic with even labels appearing along one copy of $P_{\infty}$ and odd labels along the other.

\section{REFERENCES}

[1] G. J. Chang, W.-T. Ke, D. Kuo, D. Liu, and R. Yeh, On L(d,1)-labelings of graphs, Discrete Math., 220 (2000), pp. 57-66.

[2] G. J. Chang And D. KuO, The L(2,1)-labeling problem on graphs, SIAM J. Discrete Math., 9 (1996), pp. 309-316.

[3] G. Chartrand, D.J. Erwin, F. Harary, and P. Zhang, Radio labelings of graphs, Bull. Inst. Combin. Appl., 33 (2001), pp. 77-85.

[4] J. P. Georges AND D. W. MAuro, Labeling trees with a condition at distance two, Discrete Math., 269 (2003), pp. 127-148.

[5] J. P. Georges And D. W. Mauro, Some results on the $\lambda_{j, k}$-numbers of products of complete graphs, Congr. Numer., 140 (1999), pp. 141-160.

[6] J. P. Georges And D. W. Mauro, On the size of graphs labeled with a condition at distance two, J. Graph Theory, 22 (1996), pp. 47-57.

[7] J. P. Georges and D. W. Mauro, Generalized vertex labelings with a condition at distance two, Congr. Numer., 109 (1995), pp. 141-159.

[8] J. P. Georges, D. W. Mauro, and M. I. Stein, Labeling products of complete graphs with a condition at distance two, SIAM J. Discrete Math., 14 (2000), pp. 28-35.

[9] J. P. Georges, D. W. Mauro, and M. A. Whittlesey, Relating path coverings to vertex labelings with a condition at distance two, Discrete Math., 135 (1994), pp. 103-111.

[10] J. P. Georges And D. W. Mauro, On the criticality of graphs labeled with a condition at distance two, Congr. Numer., 101 (1994), pp. 33-49.

[11] J. R. GRIGGS AND R. K. YeH, Labelling graphs with a condition at distance 2, SIAM J. Discrete Math., 5 (1992), pp. 586-595.

[12] W. K HALE, Frequency assignment: Theory and application, Proc. IEEE, 68 (1980), pp. 14971514.

[13] P. JнA, Optimal L $(2,1)$-Labeling of Kronecker Products of Certain Cycles, preprint, Universiti Multimedia Telekom, Malaysia.

[14] P. Jha, A. Narayanan, P. Sood, K. Sundaram, and V. Sunder, L(2,1)-labeling of the Cartesian product of a cycle and a path, Ars Combin., 55 (2000), pp. 81-89.

[15] D. KuO AND J. H. YAN, On L(2,1)-labelings of Cartesian products of paths and cycles, Discrete Math., to appear.

[16] D. Liu And R. YeH, On distance two labelings of graphs, Ars Combin., 47 (1997), pp. 13-22.

[17] D. SAKAI, Labeling chordal graphs: Distance two condition, SIAM J. Discrete Math., 7 (1994), pp. $133-140$.

[18] J. Van Den Heuvel, R. A. Leese, And M. A. Shepherd, Graph labeling and radio channel assignment, J. Graph Theory, 29 (1998), pp. 263-283.

[19] M. A. Whittlesey, J. P. Georges, And D. W. Mauro, On the $\lambda$-number of $Q_{n}$ and related graphs, SIAM J. Discrete Math., 8 (1995), pp. 499-506.

[20] P. Zhang, Radio labelings of cycles, Ars Combin., 65 (2002), pp. 21-32. 